\title{
A Study on Faults Diagnosis and Early-Warning Method of Tailings Reservoir Monitoring Points based on Intelligent Discovery
}

\author{
Tianyong $\mathrm{Wu}^{\mathrm{a}}$,Chunyuan Zhang ${ }^{\mathrm{b}}$, Yunsheng Zhao ${ }^{\mathrm{a}, *}$ \\ ${ }^{a}$ Faculty of Engineering, China University of Geosciences, No. 388, Lumo Road, Hongshan District, Wuhan, 430074, PR China \\ ${ }^{b}$ School of Computer Science, China University of Geosciences, No. 388, Lumo Road, Hongshan District, Wuhan, 430074, PR China
}

\begin{abstract}
The tailings reservoir is a major hazard source with high potential energy, which may cause an artificial debris flow. The stability of the tailings reservoir is extremely important to the normal operation of the mining enterprises and the safety of people's lives and property. In order to settle the problem that traditional manual monitoring is scattered, not timely, and difficult to manage, this article takes Huangmailing tailings as an example, and establishes the CMST model to optimize the network topology connection of the tailings monitoring points. BP neural network algorithm is used to discuss the intelligent discovery and early warning of the faults on-line monitoring system of tailings. In this way, the fault-points and the causes can be perceived quickly and accurately, and the risk of the tailings' safety accident can be reduced. It can be proved by the experimental results and two years stable operation of the system that BP neural network algorithm can accurately predict the value of safety monitoring data.
\end{abstract}

Keywords: Tailings; Intelligent discovery; CMST model; BP neural network; Faults diagnosis

(Submitted on April 20, 2017; Revised on June 30, 2017; Accepted on August 15, 2017)

(C) 2017 Totem Publisher, Inc. All rights reserved.

\section{Introduction}

Tailings constructed by damming intercept troughs or encirclement, and used to storage waste residue discharging from tailings or other industrial after metal or nonmetallic ore for ore sorting. The safety of the tailings is related to the safety of life and property of the state and the people. It is an important part that cannot be neglected in the process of safe production. It is of great significance to the safety of tailings to real-time monitor the operation status and provide technical support for the actual work of tailings. What's more, to discover security risks timely and send early-warning is vital to the safety of tailings as well. In recent years, with the rapid development and popularization of computer and information technology, the tailings dynamic monitoring technology gradually obtained the development. In 2011, T. L. Xu [14], etc. proposed the use of fiber and GPRS technology to transfer tailings monitoring data to ensure that the transmission system is stable and reliable. In the same year, X. Y. Wan [13], etc. used the 3S technologies combined with conventional monitoring technology for the early warning of the tailings ponds. In 2013, W. Zhang [19] described the role of the Internet in the application of public safety projects; the same year, J. F. Zhang [18], etc. proposed lab view based tailings information monitoring and management system. Based on three-dimensional GIS, L. Huang [4], etc. proposed a tailings reservoir monitoring and early warning system technical program, and monitored the regional tailings dam disaster. In 2014, Y. Z. Gao [2], etc. established accurate remote sensing interpretation by using high-resolution remote sensing images, which provides scientific basis for the supervision and management of tailings. In the same year, based on high-resolution satellite data, L. F. Zhang [17], etc. carried out the northern Liaoning coal mine ecological environment monitoring. Based on OpenGL and GIS technology, W. J. Liao [7], etc. established the three-dimensional real-time monitoring and early warning system of tailings. In 2015, S. Y. Dong and Q. M. Li [1] put forward the structure framework of the digital system, and the realization methods of sub-system. In the same year, H. M. Zhou [16], etc. analyzed the present development and research situation of safety online-monitoring system for tailings pond. 
M. Necsoiu and G. R. Walter [9] used satellite radar interferometry to monitor the tailings sedimentation. Q. S. Huang [5], etc. through the camera monocular vision to calculate the water line pixel coordinates and the actual length of the beach geometric conversion formula, and carry out real-time monitoring of dry beach length. In 2016, L. G. Wang [12] applied Vibrating wire sensor to the online monitoring of tailings. In the same year, S. G. Sun [10], etc. through the three-dimensional digital simulation and the establishment of MIDAS program to monitor the stability of the tailings. Y. Huang [6] proposed a differential evolution filtering algorithm and applied it to the monitoring information processing of tailings leaking line. D. C. Huang and S. C. Xie [3] applied BP neural network in monitoring tailings dam settlement. In 2017, J. Wang [11], etc. proposed a fractional gradient descent method for the BP training of neural networks, and the monotonicity and weak (strong) convergence of the proposed approach are proved in detail. For various reasons, the online monitoring network is vulnerable to damage and lead to network paralysis, so that the safety situation of the tailings go out of the safety management personnel's control. Under severe weather conditions, if the field troubleshooting is carried out, the physical safety of the technician cannot be guaranteed. Therefore, in view of the problems for difficulty fault diagnosis existing in the safety monitoring network of tailings, it is of great significance to discovery intelligent fault diagnosis method and design intelligent fault diagnosis and early warning system of tailings.

\section{CMST Model and BP Neural Network Algorithm}

CMST (Constrained Minimum Spanning Tree) model is usually applied to centralized network and tree structure optimization processing. In the case of a tree network, it is assumed that there are $n$ nodes in the network, where node $\mathrm{M}$ is the central node and the other nodes transmit data to the central node M. The constraint condition is that the load does not exceed the given value, and the path length does not exceed the given value. The function of CMST refers to finding the smallest spanning tree consisting of known node sets under certain constraints [8].

\subsection{CMST Optimization Algorithm Model}

- Definition 1 Node set: A set of interconnected nodes that are not connected to the central node M.

- Definition 2 The distance between nodes: The minimum cost of direct connection between two node sets.

- Definition 3 Current Consider Nodes: During the optimization process, the nodes, which are considered to be connected by other nodes or not, are regarded as $\mathrm{CCN}$.

- Definition 4 Further Consider Nodes: A set of interconnected nodes that are not connected to the central node M during the optimization process, denoted as FCN.

- Assumption 1: If the capacity of each link is the same, the star network must meet the constraint conditions.

- Algorithm ideas: Starting from the farthest node, the nearest node is set into a set of nodes. In the premise of the constraints, the node is connected as much as possible. Otherwise the node set is connected with the center node to form a sub tree. The specific process is shown in Figure 1.

At least one node or set of nodes will be deleted from further considerations in each cycle of Step 3 to Step 9 of the algorithm, so the loop is limited when the input is deterministic. Similarly, each cycle from Step 5 to Step 7 will merge a node or node set to the $\mathrm{CCN}$ in the case of a constraint, so the loop is limited as well.

According to assumption 1, each node is satisfied with the constraint conditions at the beginning, and Step 7 of the algorithm makes sure that the new CNN in Step 8 is also satisfied with the constraint conditions. What's more, the sub tree of connected with the central node $\mathrm{M}$ is satisfied with the constraint conditions as well, so the results are feasible. If the input is given, the process is unique and the result is unique. If the output is deterministic, the star network is feasible, the algorithm will stop after a finite number of calculations, and the result is unique and feasible.

\subsection{Theories of BP Neural Network Algorithm}

BP neural network algorithm is a supervised learning process. The input and output samples are used to train the network, and weights have been adjusted during the training process. It makes the adjustment of the weights proportional to the gradient of the errors. In this way, errors can be reduced to meet the needs of the application [18]. BP neural network consists of input layer, hidden layer and output layer, and its network structure is shown in Figure 2.

In the BP neural network, the unipolar sigmoid function is usually used as the transfer function:

$$
f(x)=\frac{1}{1+e^{-x}}
$$




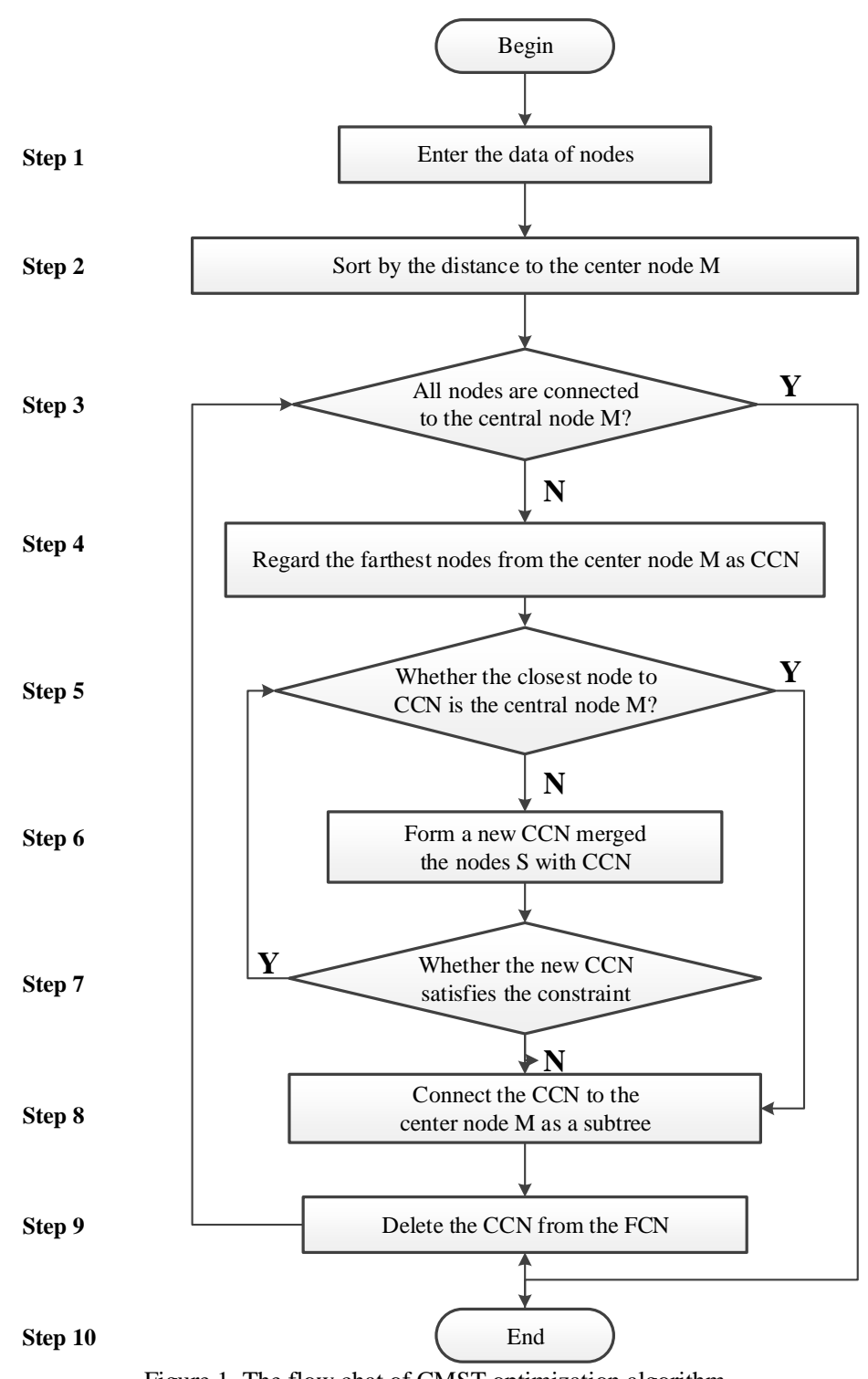

Figure 1. The flow chat of CMST optimization algorithm

In practice, bipolar sigmoid functions can also be used according to the need:

$$
f(x)=\frac{1-e^{-x}}{1+e^{-x}}
$$

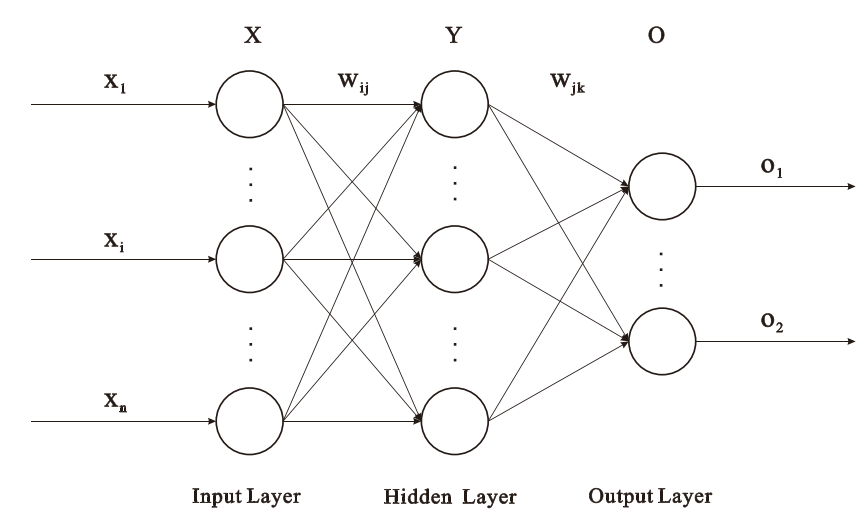

Figure 2. BP neural network structure 
The mathematical relationship among the input layer $X$, the hidden layer $Y$ and the output layer $O$ in the BP neural network is as follows:

For the hidden layers, there are:

$$
\mathrm{y}_{j}=f\left(\sum_{i=0}^{n} \omega_{i j} x_{i}+\theta_{i}\right) i=1,2, \ldots, m ; j=1,2, \ldots, m
$$

For the output layers, there are:

$$
\mathrm{O}_{k}=f\left(\sum_{j=0}^{m} \omega_{j k} y_{i}+\theta_{j}\right) j=1,2, \ldots, l ; k=1,2, \ldots, l
$$

In the formula (3) and (4), $\omega$ is the connection weight between nodes, and $\theta$ is the bias value of the neuron.

In the initial BP network training stages, The network output $O$ and the expected output $T$ often have an error Err, for the error of the output layer node $k$ :

$$
\operatorname{Err}_{k}=O_{k}\left(1-O_{k}\right)\left(T_{k}-O_{k}\right)
$$

For the error of the hidden layer node $\mathrm{j}$ :

$$
\operatorname{Err}_{j}=O_{j}\left(1-O_{j}\right) \sum_{k} E r r_{k} \omega_{j k}
$$

In the case of a learning rate of $l$, the formula of updating weights is as follows:

$$
\omega_{i j}^{\prime}=\omega_{i j}+(l) \operatorname{Err}_{j} O_{i}
$$

Similarly, the formula of updating bias values is as follows:

$$
\theta_{j}^{\prime}=\theta_{j}+(l) E r r_{j}
$$

If $\operatorname{Err}_{i}>\operatorname{Err}_{0}$ (Erro is a pre-set error), the network continues to the next round of learning and adjust the weight, otherwise the network stops learning. After the end of the learning, the network consisting of the $\omega_{i j}$ weight and the $\theta$ bias can achieve the desired output within the error range set by Erro.

\section{The Design of Faults Intelligent Discovery Algorithm}

\subsection{The Optimization of Monitoring Points Network Topology Structural}

In theory, the star topology is the optimal network topology, but the cost is too high when taking the difficulty of construction into consideration. This paper integrates the efficiency of network transmission with the cost, and proposes a method to optimize the topology of the monitoring point network based on the CMST model. Taking the TengJiachong tailings in Hubei Province Huang Mailing Phosphorus Chemical Co. Ltd. as an example, if it follows the star network topology, its topology is shown in Figure 3.

According to Figure 3, there are 10 nodes in the TengJiachong tailings, in which the switching centre is the central node $\mathrm{M}$ and the network load of the node $i$ is expressed by $\alpha i$. The network load of each node is shown in Table 1 . The link cost (expressed by the distance of the fibre) that the nodes may be connected to each other is shown in Table 2. The constraint condition is that a link network load does not exceed 5. 


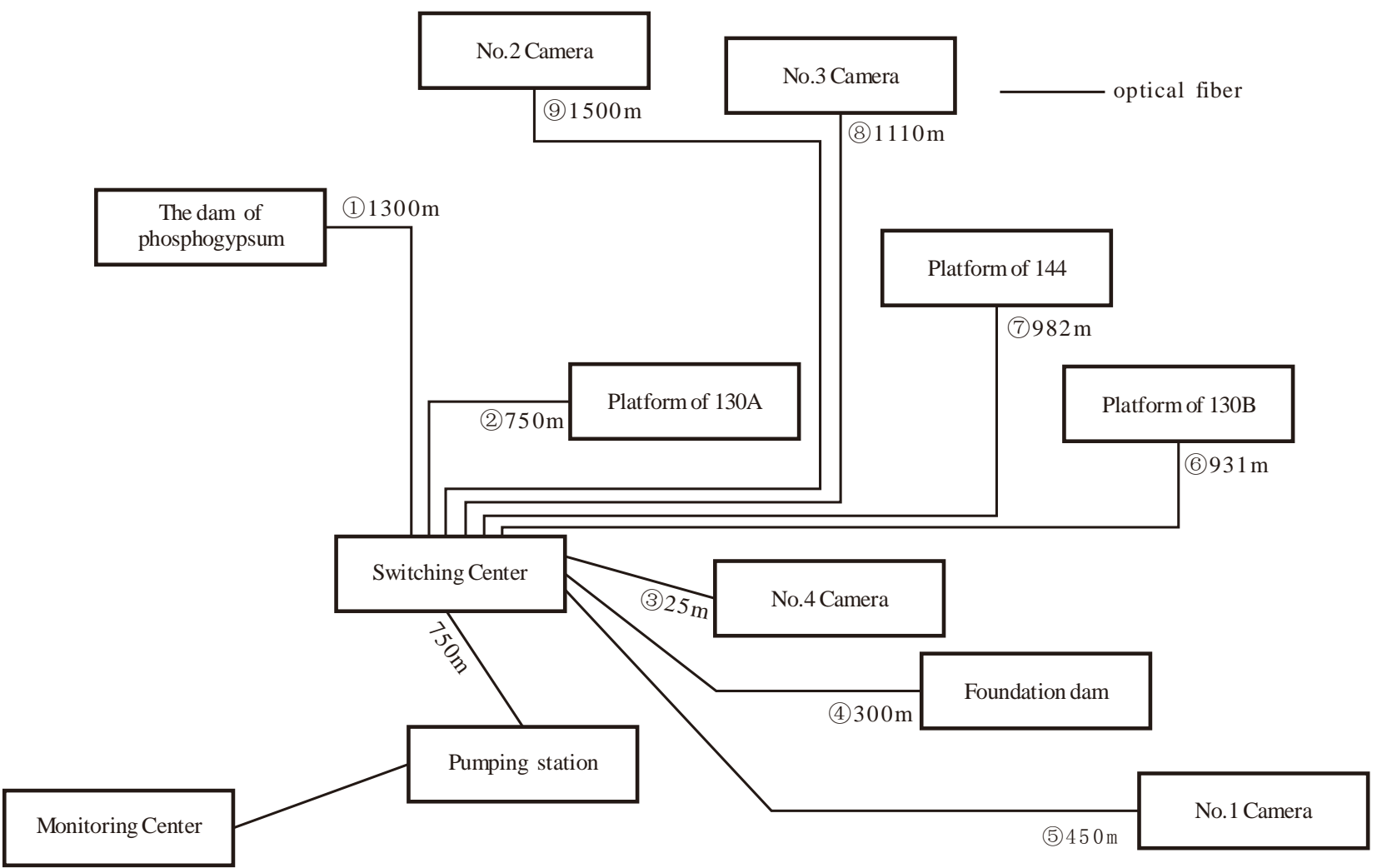

Figure 3. The star network topology of the tailings of Hubei Huangmailing phoschemical industry CO., LTD

Table 1. Network load table for each node

\begin{tabular}{|l|c|l|}
\hline \multicolumn{1}{|c|}{ Node Number } & Network Load $(\alpha \mathrm{i})$ & \multicolumn{1}{c|}{ Remarks } \\
\hline (1)The dam of Phosphogypsum & 3 & GPS Base station $\times 1$, Wireless bridge $\times 2$ \\
\hline (2)Platform of 130A & 2 & GPS $\times 1$, Serial Device Serve $\times 1$ \\
\hline (3)No.4 Camera & 1 & Spherical camera $\times 1$ \\
\hline (4)Foundation dam & 2 & GPS $\times 1$, Wireless bridge $\times 1$ \\
\hline (5)No.1 Camera & 1 & Gun camera $\times 1$ \\
\hline (6)Platform of 130B & 1 & GPS $\times 1$ \\
\hline (7)Platform of 144 & 2 & GPS $\times 1$, Wireless bridge $\times 1$ \\
\hline (8)No.3 Camera & 1 & Spherical camera $\times 1$ \\
\hline (9)No.2 Camera & 2 & Spherical camera $\times 1$, Serial Device Serve $\times 1$ \\
\hline
\end{tabular}

Note: Assume that the data traffic of each network device is the same.

Table 2. The Link Cost Matrix between the Nodes

\begin{tabular}{|c|c|c|c|c|c|c|c|c|c|c|}
\hline & $\mathrm{M}$ & 1 & 2 & $(3$ & 4 & 5 & 6 & 7 & (8) & (9) \\
\hline $\mathrm{M}$ & - & 1300 & 750 & 25 & 300 & 450 & 931 & 982 & 1110 & 1500 \\
\hline 1 & 1300 & - & - & - & 1600 & - & - & 2282 & - & - \\
\hline$(2)$ & 750 & - & - & 725 & 750 & 850 & 181 & 270 & 360 & 750 \\
\hline 3 & 25 & - & 725 & - & 275 & 425 & 906 & 957 & 1085 & 1475 \\
\hline$(4)$ & 300 & 1600 & 750 & 275 & - & 150 & 300 & 350 & - & - \\
\hline 5 & 450 & - & 850 & 425 & 150 & - & 400 & 450 & - & - \\
\hline$(6$ & 931 & - & 181 & 906 & 300 & 400 & - & 50 & 178 & 568 \\
\hline 7 & 982 & 2282 & 270 & 957 & 350 & 450 & 50 & - & 128 & 518 \\
\hline 8 & 1110 & - & 360 & 1085 & - & - & 178 & 128 & - & 390 \\
\hline$(9$ & 1500 & - & 750 & 1475 & - & - & 568 & 518 & 390 & - \\
\hline
\end{tabular}

According to the link cost matrix between each node, node 9 is the farthest away from the central node $\mathrm{M}$ in the available links. So taking node 9 as the CCN, we find node 8 can be merged into a new CCN1 through Step 5 to Step 7 . The link cost at this time is 3 and satisfies the constraint conditions. Then the second cycle begins from Step 5, and the node 7 is merged into a new CCN2. The link cost at this time is 5 and satisfies the constraint conditions. And then the third cycle begins from Step 5, and the node 6 is merged into a new CCN3. The link cost at this time is 6 and does not meet the constraint conditions. 
So jumping out of the cycle, the CCN2 is connected with the central node M as Step 8 performed. The resulting sub tree M7-8-9 is shown in Figure 4-a.

From further consideration, FCN1 takes node 1 as $\mathrm{CCN}$, and the first cycle starts from Step 5. For the reason that the nearest node from the $\mathrm{CCN}$ is the central node $\mathrm{M}$, the loop is broken, and the $\mathrm{CCN}$ is connected with the central node $\mathrm{M}$ as Step 8 implemented. The process of resulting sub tree M-1 is shown in Figure 4-b.

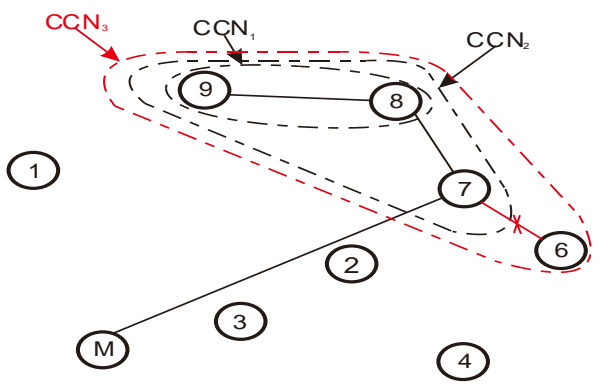

(a)

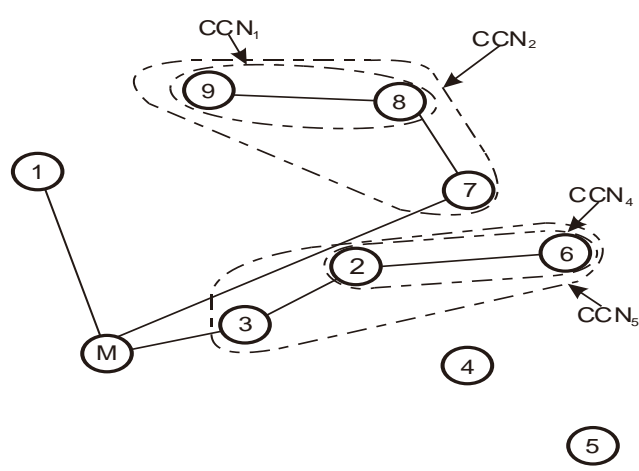

(c)

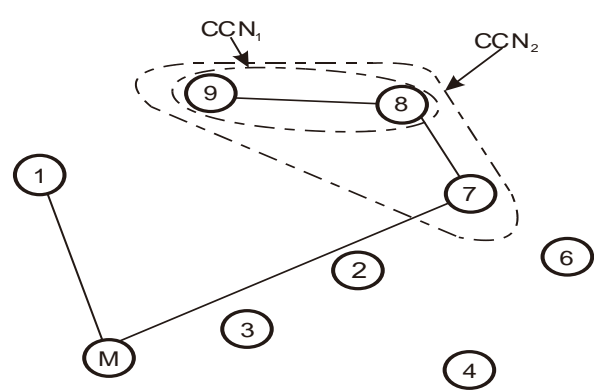

(b)

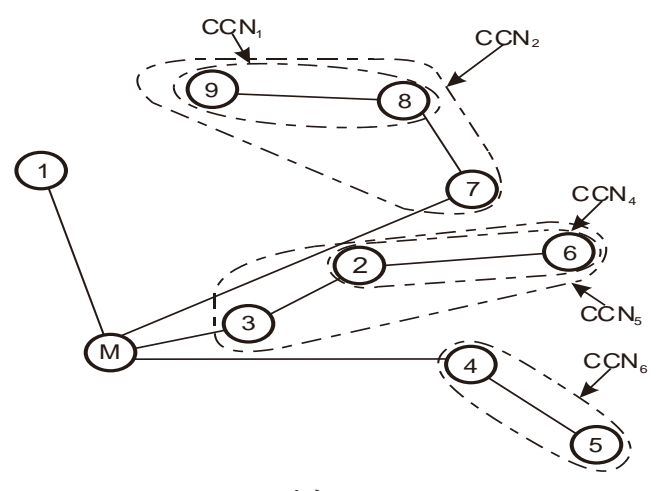

(d)

Figure 4. Process diagram of network topology optimization algorithm for monitoring points of the tailings

From further consideration, FCN2 takes the node 6 as CCN, and the first cycle starts from Step 5. Firstly, node 2 is merged into the new CCN4, and the link cost is 3, which meets the need of the constraint conditions. Secondly, the second cycle begins from Step 5, and the node 3 is merged into a new CCN5. The link cost at this time is 4 and meets the need of the constraint conditions. Thirdly, the third cycle starts from Step 5, the cycle is broken for the reason that the nearest node from the CCN5 is the central node M. As implemented in Step 8, the CCN5 is connected to the central node M. The forming process of the resulting sub tree M-3-2-6 is shown in Figure 4-c.

From further consideration, $\mathrm{FCN}_{3}$ takes node 5 as $\mathrm{CCN}$, and the first cycle begins from Step 5. Firstly, we find node 4 can be merged into CCN6, and the link cost at the time is 3, which satisfies the constraint conditions. Secondly, the second cycle begins from Step 5, the nearest node from the CCN6 is the central node M. So jumping out of the cycle, the CCN6 is connected to the central node M as carried out in Step 8. The resulting sub tree M-4 -5 is shown in Figure 4-d.

\subsection{The Design of Faults intelligent Diagnosis and Early Warning System}

The faults intelligent diagnosis and early warning system is based on BP neural network. It consists of four parts: BP neural network training module, the acquisition and transformation module of network state information, faults diagnosis analysis module and fault early warning module. The structure of the system is shown in Figure 5.

In order to obtain the trained BP network, the BP neural network training module trains the existing monitoring data in the security monitoring database through the learning machine. Then, the trained BP network is used to predict the amount of the data reception of the central node $\mathrm{M}$. The value of the data reception is marked as $E_{0}$. 


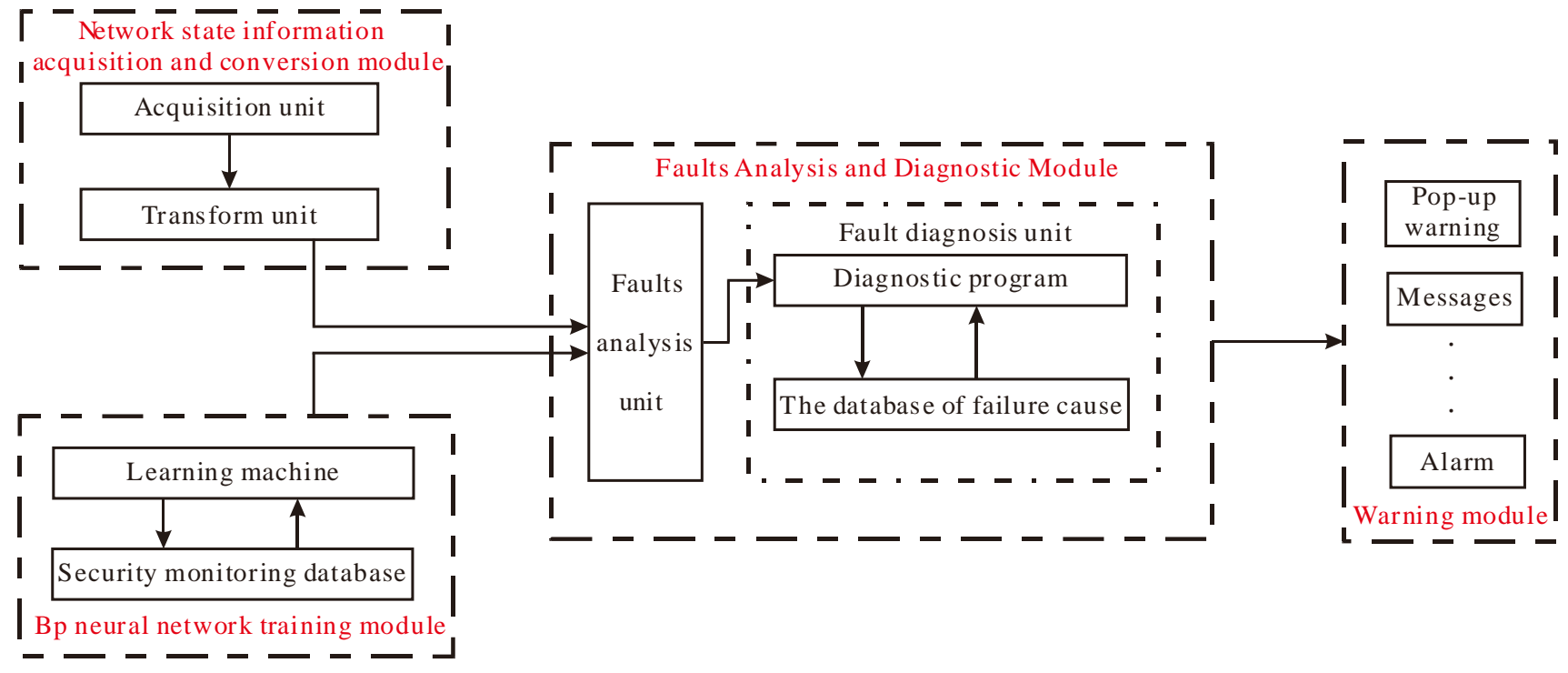

Figure 5. Fault diagnosis and early warning system architecture based on BP neural network

The amount of the monitoring data reception is obtained by the network information acquisition and transformation module. The value of the monitoring data reception is marked as $E$. Then, the analysis unit determines whether $\Delta E=E 0-E$ is within error permissibility. If $\Delta E$ exceeds the error permissibility, the diagnostic program sends UDP packets to the network equipment of each monitoring point, and records the results of corresponding return and network delay. After that, the diagnostic program matches the results to the faults reason database. Finally, the faults diagnosis analysis module informs the security manager of the faults diagnosis results. The system flowchart is shown in Figure 6.

\section{Experimental Results and Analysis}

\subsection{Brief introduction of research area}

May 1992, the former Ministry of Chemical Industry Mining Planning and Design Institute completed the design of the TengJiachong tailings pond in Huangmailing Phosphorus Chemical Co., Ltd. of Hubei Province. The design capacity of the tailings is 1410.8 million cubic meters. The final crest elevation is 177 meters. The total height of the dam is 1072 meters. The length of the dam is 610 meters. The service life of the tailings is 23 years. The TengJiachong tailings pond is a valleytype tailings reservoir built in upstream construction method. Its initial dam is a permeable rock fill dam. The crest elevation of the initial dam is 70 meters. The length of the initial dam is 172 meters. The height of the initial dam is 30 meters. As to sub-dams, their height is 1 meter. The slope ratio of the tailing dam is 1:4, and the final design elevation is 177 meters [15].

In the pre-project, the network topology is a mesh topology. Once one of the nodes fails, the associated network nodes will be affected, and the data can't be sent to the acquisition centre. In order to improve the safety performance of the equipment and ensure the efficiency of data transmission, the second phase of the project will change wireless transmission to optical fibre transmission. The pre-bridge equipment will still be left in the monitoring point as a backup line.

The distance between the TengJiachong tailings dam and the highest point of the mining site is 1 kilometre. The straight line distance between the highest point and the monitoring centre is about 2 kilometres. Taking the actual location and topographic factors into consideration, the project chose to collect the data by using kinds of sensors, to transmit the data through the wireless network. The goal of the project is to realize the safety monitoring of the tailings dam. The design of tailings' wireless transmission network is shown in Figure 7. Inside the dashed box is the wireless transmission station.

Since monitoring points are numerous and widely distributed, the signal is often blocked by mountains and trees. In order to solve this problem, the wireless bridge is often erected at high ground in the middle of the monitoring points to transfer the signal. However, it makes the number of network nodes and the probability of failures increased. Because wireless bridges must be erected in the air, they are easily damaged by lightning. Therefore, different kinds of failures have occurred after the initial project put into operation. 


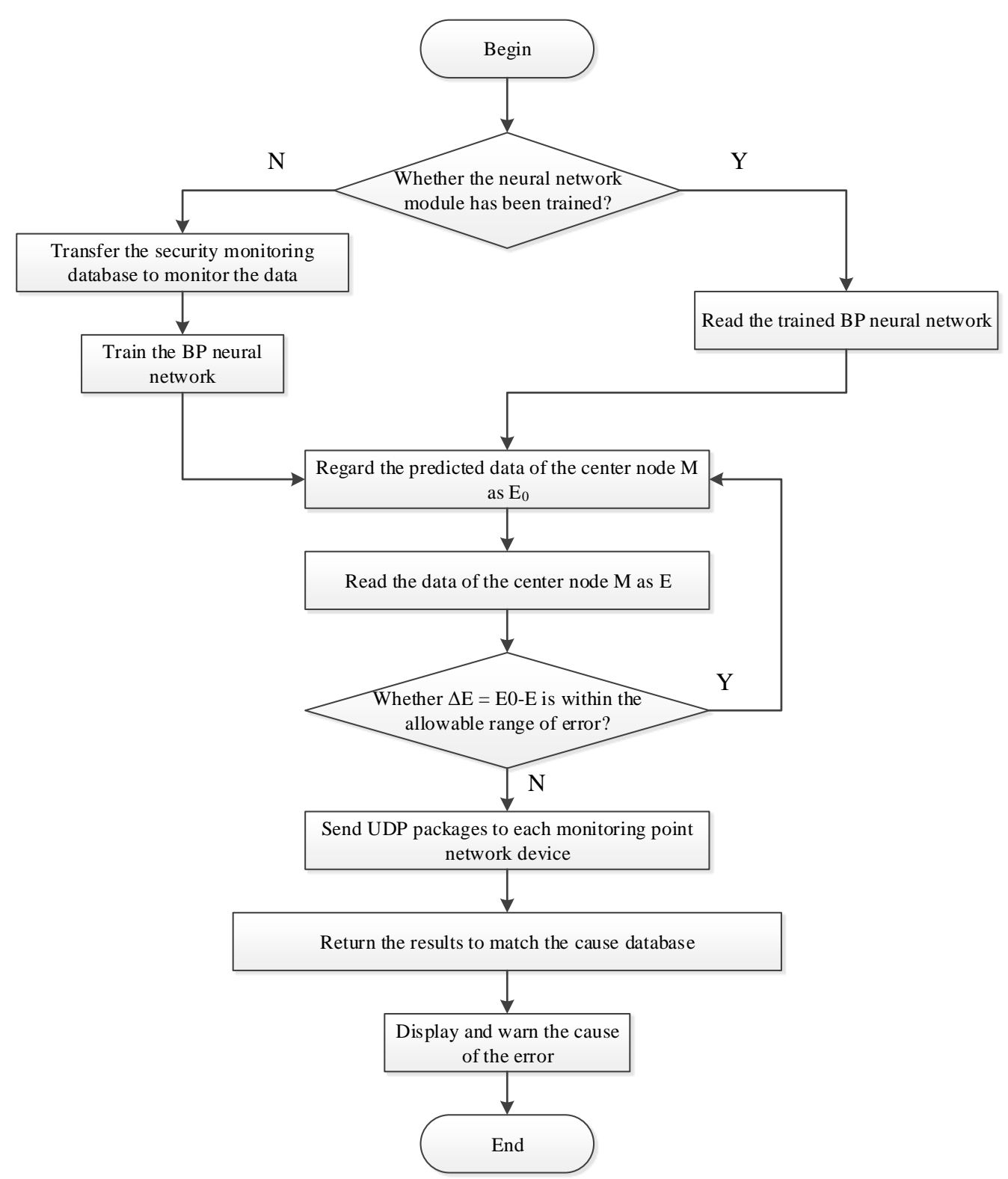

Figure 6. Flowchart of intelligent fault diagnosis and early warning system based on BP neural network

\subsection{Result of Monitoring Network Topology Optimization}

In summary, through the optimization algorithm based on CMST model, we can get the network topology satisfying the constraint condition of the minimum fiber routing cost under reasonable load distribution. The total link cost is 3181 , and its network topology is shown in Figure 8.

Compared with the wireless transmission network design of the early-stage project and the star network topology, the network topology optimized by the CMST model algorithm has many advantages. This network topology can effectively control the cost of wiring, and reduce a large number of the use of wireless transmission equipment. In this way, the problem of equipment's lightning damage can be solved effectively and the stability of the network transmission can be ensured.

\subsection{Process of Faults Intelligent Discovery}

As to TengJiachong tailings, the data for the input layer is the data transmission amount of each monitoring point. The data for the out layer is the data reception amount of the central node $\mathrm{M}$. Then, we call the security monitoring system database of TengJiachong tailings reservoir for the monitoring data transmission of each monitoring point in the period $\mathrm{T}=5 \mathrm{~min}$ (part as shown in Table 3), and import the data into MATLAB through Code 1. 


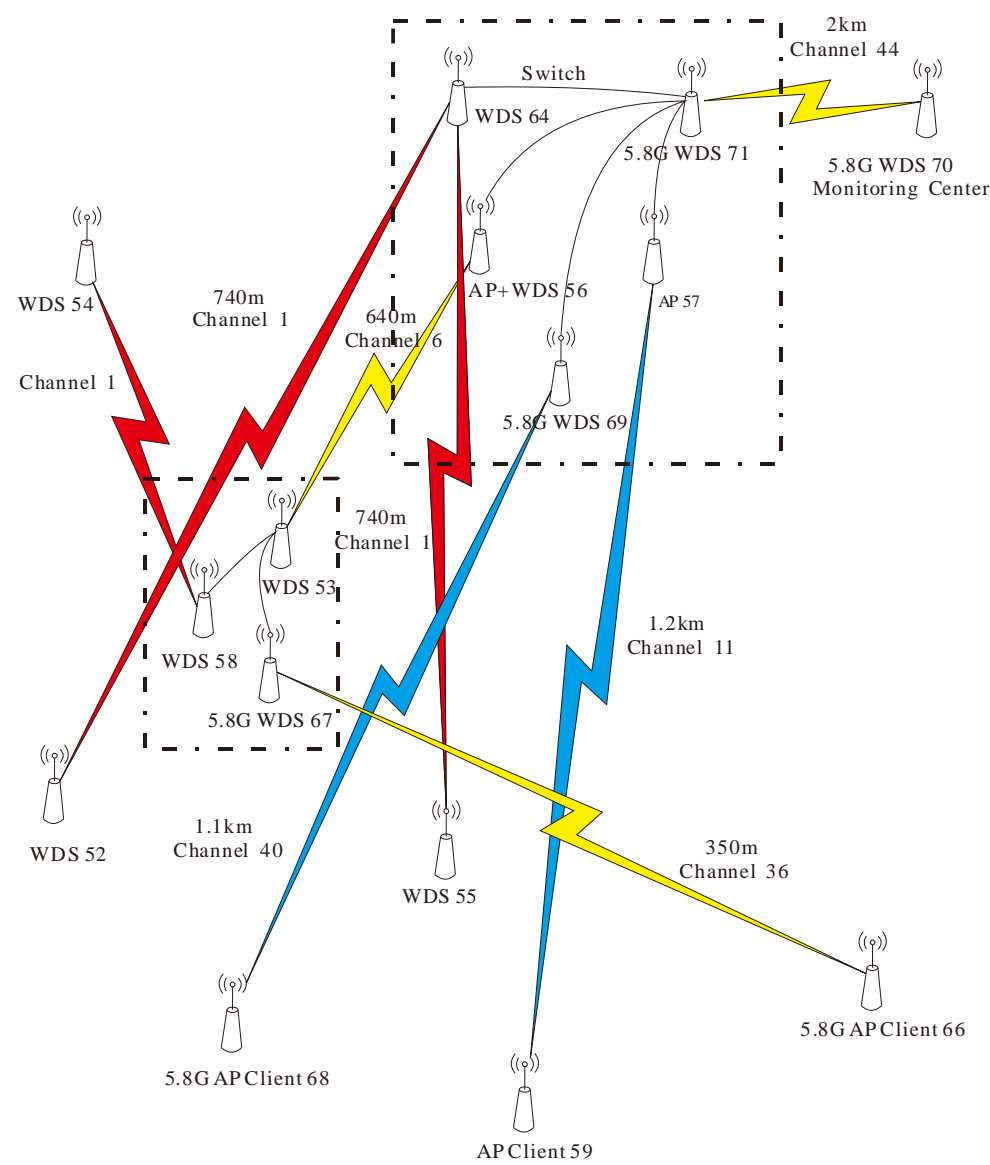

Figure 7. The topology of tailings library wireless transmission network

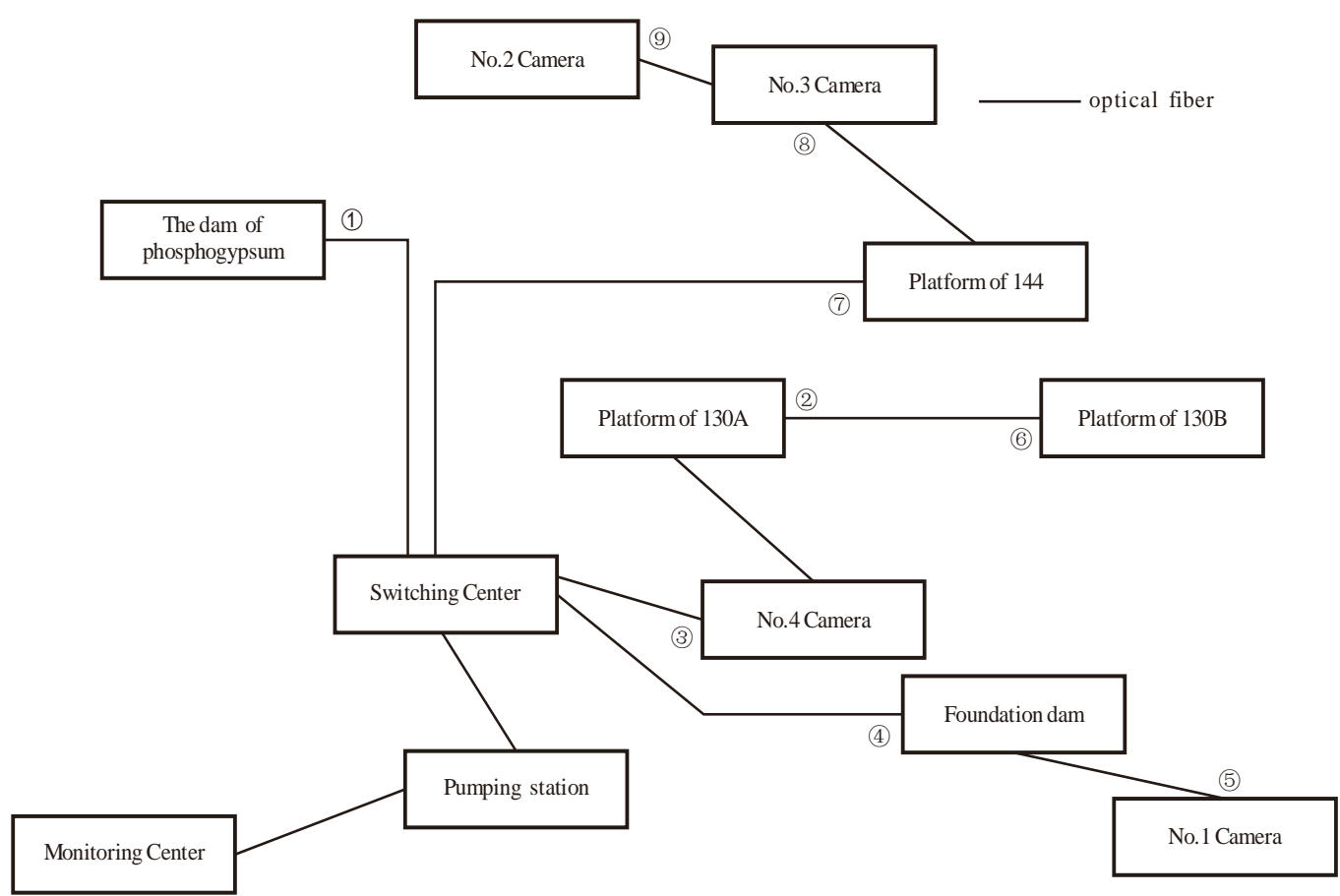

Figure 8. Results of the monitoring network topology optimization of the tailings 
Code 1: function [data, number] = getdata (' Table 3 Monitoring data transmission amount of each monitoring point in the TengJiachong tailings reservoir ')

[number , ] =xlsread (xlsfile,'A3:A2012');

[x1, ] $=x$ xlsread (xlsfile,'B3:B2012');

$[\mathrm{x} 2, \sim]=x 1 s r e a d(x 1 s f i l e, ' C 3: C 2012 ')$;

[x3, ] =xlsread (xlsfile,'D3:D2012');

[x4, ]=xlsread (xlsfile,'E3:E2012');

[x5, ] $=x$ lsread (xlsfile,'F3:F2012');

[x6, ] =xlsread (xlsfile,'G3:G2012');

[x7, ]=xlsread (xlsfile,'H3:H2012');

[x8, ] $=x l s r e a d ~(x l s f i l e, ' I 3: I 2012 ')$;

[x9, ] $=$ xlsread (xlsfile,'J3:J2012');

[M, ]=xlsread (xlsfile,'K3:K2012');

data $=[\mathrm{x} 1, \mathrm{x} 2, \mathrm{x} 3, \mathrm{x} 4, \mathrm{x} 5, \mathrm{x} 6, \mathrm{x} 7, \mathrm{x} 8, \mathrm{x} 9, \mathrm{M}]$;

end

Table 3. Monitoring data transmission amount of each monitoring point in the TengJiachong tailings reservoir

\begin{tabular}{|c|c|c|c|c|c|c|c|c|c|c|}
\hline \multicolumn{10}{|c|}{ data transmission amount } & \multirow{2}{*}{\begin{tabular}{|c|c|c}
$\begin{array}{c}\text { data } \\
\text { reception } \\
\text { amount }\end{array}$ \\
$\mathrm{M}$ \\
\end{tabular}} \\
\hline & (1) & (2) & (3) & (4) & (5) & (6) & (7) & (8) & (9) & \\
\hline $\mathrm{T} 1$ & 4573.8 & 9783.7 & 13009.4 & 4399.2 & 12970.8 & 4487.7 & 4408.4 & 13732.2 & 19732.9 & 87098.1 \\
\hline $\mathrm{T} 2$ & 4860.6 & 9378.4 & 13702.3 & 4603.7 & 13049.9 & 4209.4 & 4378.6 & 13213.1 & 19083.6 & 86179.6 \\
\hline T3 & 4798.3 & 9583.7 & 13145.2 & 4478.8 & 12864.7 & 4389.2 & 4579.2 & 13579.4 & 19537.1 & 86955.6 \\
\hline $\mathrm{T} 4$ & 4687.6 & 9473.6 & 13347.8 & 4037.7 & 13156.2 & 4109.3 & 4281.5 & 13689.7 & 19934.9 & 86718.3 \\
\hline T5 & 4978.9 & 9564.6 & 13564.5 & 4654.6 & 13365.4 & 4234.6 & 4321.5 & 13879.9 & 19564.6 & 88128.6 \\
\hline T6 & 4352.1 & 9126.1 & 13565.2 & 4234.2 & 12875.3 & 4552.1 & 4210.8 & 13215.4 & 19423.1 & 85554.3 \\
\hline $\mathrm{T} 7$ & 4895.6 & 9642.8 & 13246.7 & 4531.2 & 12964.3 & 4359.7 & 4119.4 & 13147.8 & 19827.8 & 86735.3 \\
\hline $\mathrm{T} 8$ & 4811.3 & 9246.5 & 13289.1 & 4154.8 & 13276.4 & 4167.8 & 4701.3 & 13642.4 & 19830.7 & 87120.3 \\
\hline T9 & 4714.6 & 9478.1 & 13765.4 & 4312.6 & 13531.7 & 4296.7 & 4639.8 & 13479.2 & 19688.8 & 87906.9 \\
\hline T10 & 4434.9 & 9603.5 & 13673.7 & 4598.7 & 13481.2 & 4516.3 & 4267.9 & 13197.6 & 19381.7 & 87155.5 \\
\hline
\end{tabular}

Then, normalizing the imported data according to Code 2 (part as shown in Table 4):

Table 4. Normalized results of monitoring data transmission amount of each monitoring point

\begin{tabular}{|c|c|c|c|c|c|c|c|c|c|c|}
\hline & \multicolumn{9}{|c|}{ data transmission amount } & \multirow{2}{*}{$\begin{array}{c}\begin{array}{c}\text { data } \\
\text { reception } \\
\text { amount }\end{array} \\
\mathrm{M} \\
\end{array}$} \\
\hline & (1) & (2) & (3) & (4) & (5) & (6) & (7) & (8) & (9) & \\
\hline $\mathrm{T} 2$ & 0.8113 & 0.3837 & 0.5197 & 0.9175 & 0.2777 & 0.2261 & 0.4454 & 0.0892 & 0 & 0.2429 \\
\hline T3 & 0.7119 & 0.6959 & 0.1796 & 0.7150 & 0 & 0.6321 & 0.7902 & 0.5895 & 0.5327 & 0.5443 \\
\hline T6 & 0 & 0 & 0.7352 & 0.3185 & 0.0159 & 1 & 0.1571 & 0.0923 & 0.3988 & 0 \\
\hline $\mathrm{T7}$ & 0.8671 & 0.7857 & 0.3139 & 0.8000 & 0.1493 & 0.5655 & 0 & 0 & 0.8742 & 0.4588 \\
\hline T8 & 0.7326 & 0.1831 & 0.3700 & 0.1898 & 0.6172 & 0.1321 & 1 & 0.6756 & 0.8776 & 0.6083 \\
\hline T9 & 0.5783 & 0.5353 & 1 & 0.4456 & 1 & 0.4232 & 0.8943 & 0.4527 & 0.7109 & 0.9139 \\
\hline $\mathrm{T} 10$ & 0.1321 & 0.7260 & 0.8787 & 0.9094 & 0.9243 & 0.9192 & 0.2552 & 0.0680 & 0.3502 & 0.6220 \\
\hline
\end{tabular}

Code 2: function normal $=$ normalization $(\mathrm{x})$

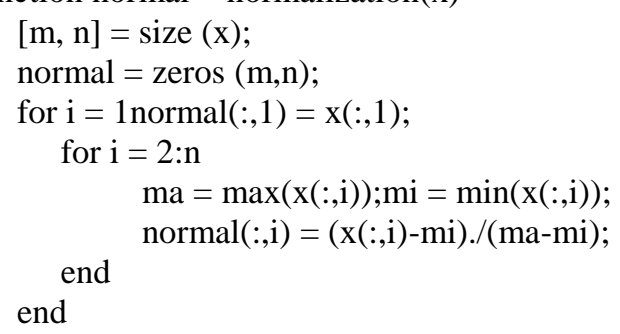


There are 9 monitoring points in the TengJiachong tailings. Meanwhile, the data reception of the central node $\mathrm{M}$ during a time period is the condition to determine the failure of monitoring points. Therefore, the BP neural network module has 9 input nodes and 1 output node. A three-tier structure is used in the BP neural network module which consist of input layer, hidden layer and output layer. The number of neurons in the hidden layer is determined by the convergence rate of the neural network under the different number of neurons. In the model, the minimum error is 0.0000000001 , the maximum step is 500 and the learning rate is 0.05 . The experimental results are shown in Table 5.

Table 5. Comparison of the convergence rates of neural networks under different number hidden neurons

\begin{tabular}{|c|c|c|c|c|c|c|c|c|c|}
\hline Hidden neurons number & 1 & 2 & 3 & 4 & 5 & 6 & 7 & 8 & 9 \\
\hline Learning step (step) & 350 & 246 & 222 & 230 & 300 & 290 & 400 & 260 & 237 \\
\hline Convergence time (seconds) & 11 & 7 & 5 & 6 & 9 & 8 & 9 & 7 & 6 \\
\hline
\end{tabular}

According to Table 5, when the hidden layer has 3 neurons, the BP neural network module has fastest convergence rate. Therefore, this article set 3 as the number of hidden layer neurons and establishes the BP neural network shown in Figure 9 to analysis the monitoring data.

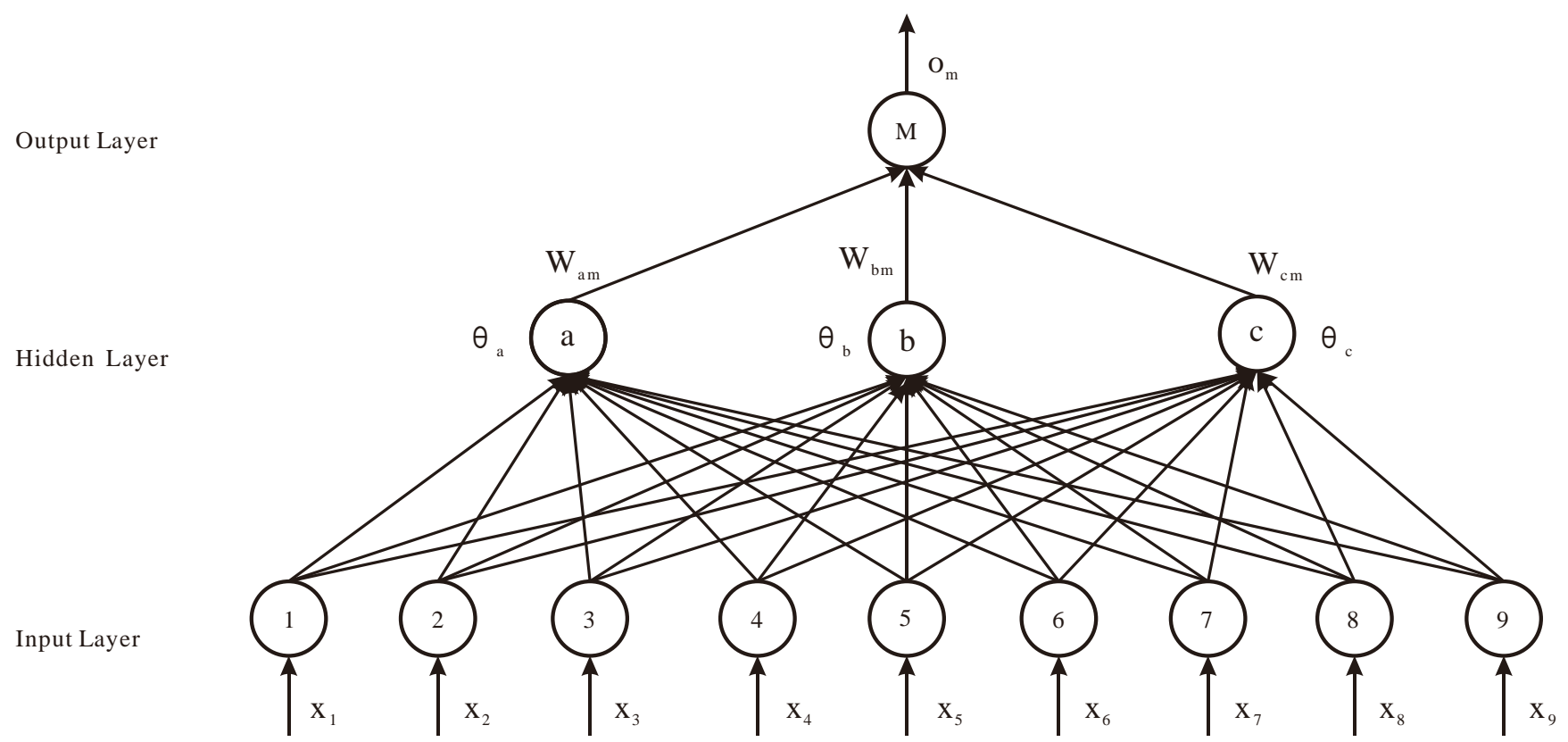

Figure 9. BP neural network of the monitoring data transmission amount of each monitoring point

Firstly, the first 2000 sets of data in Table 3 are used as training data, and the data in group 2001 to 2010 are used as test data. Setting $\operatorname{Err}_{0}=0.0000000001$, learning rate $l=0.05$, and the maximum number of iterations $=500$, the BP neural network training chart (shown in Figure 10) and the training simulation chart (Figure 11) can be obtained according to Code 3.

Code 3: input_train $=\operatorname{data}(1: 2000,1: 9)$;

output_train = data $(1: 2000,10)^{\prime}$;

input_train = data $(1: 2000,1: 9)^{\prime}$;

input_train $=$ data $(1: 2000,1: 9)$;

input_train $=$ data $(1: 2000,1: 9)^{\prime}$;

input_test $=$ data $(2001: 2010,1: 9)^{\prime}$;

[inputn,inputps] = mapminmax(input_train);

[outputn,outputps] = mapminmax(output_train);

net = newff(inputn, outputn,3);

net.trainParam.epochs $=500$;

net.trainParam.1 = 0.05;

net.trainParam.Err0 $=0.0000000001$;

net $=\operatorname{train}($ net,inputn, outputn); 


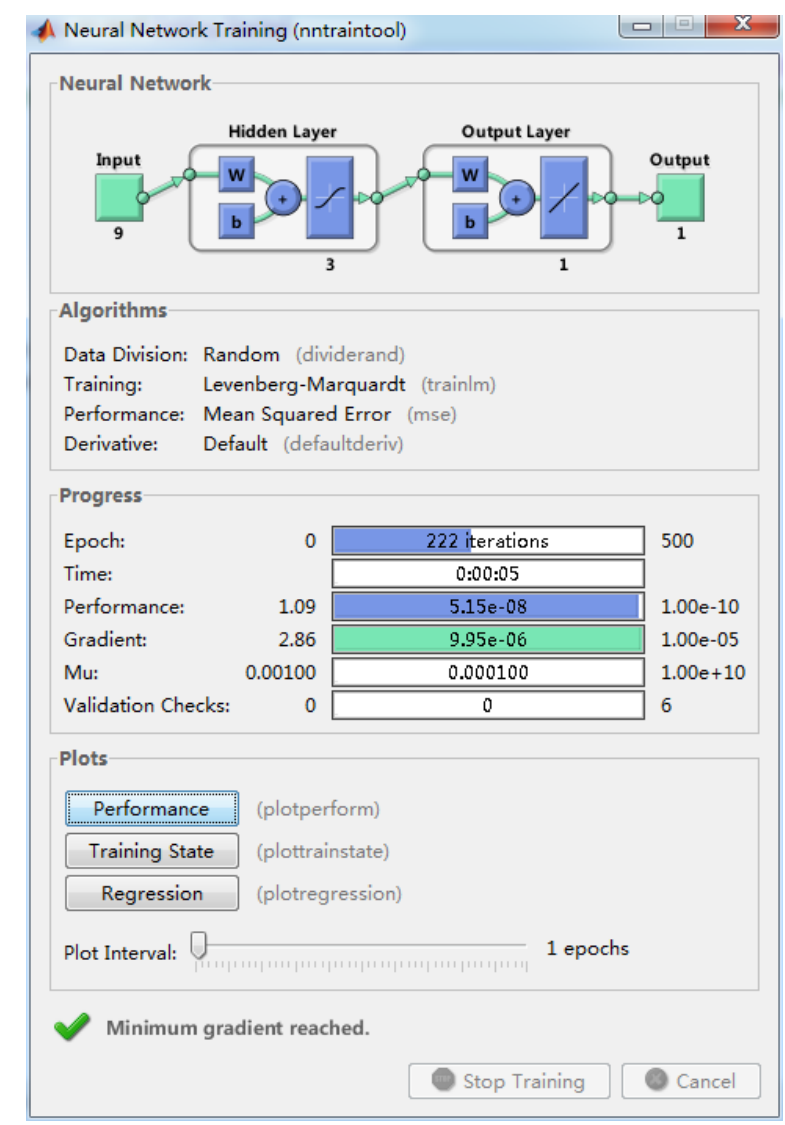

Figure 10. BP neural network training chart

It can be seen from Figure 11, the test data curve and the validation data curve are basically consistent to the training data curve. Therefore, the training is feasible. The code 4 is used to predict the data in group 2001 to 2010 (shown in Table 6).

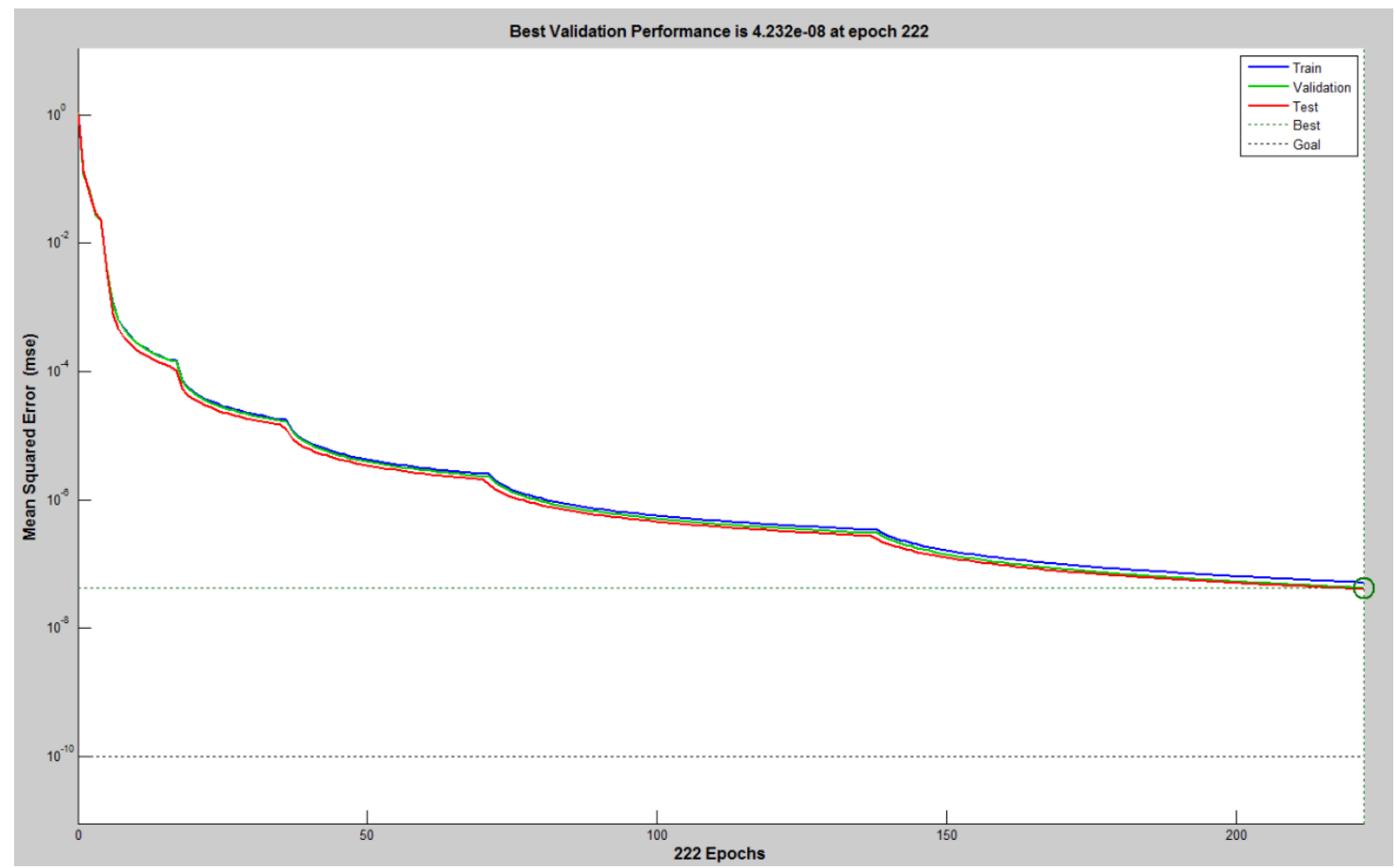

Figure 11. BP neural network training chart 
Code 4: inputn_test = mapminmax ('apply', input_test, inputps); an $=\operatorname{sim}($ net, inputn_test);

The results of code 4 are shown as follow:

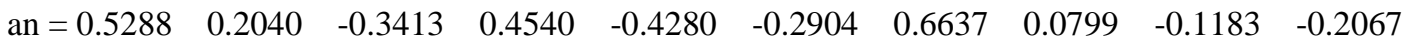

Then, using Code 5 to predict the data.

Code 5: BPoutput = mapminmax ('reverse', an, outputps);

The results are shown as follow:

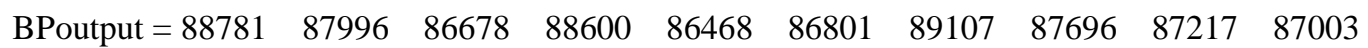

Table 6. Monitoring data from Group 2001 to 2010

\begin{tabular}{|c|c|c|c|c|c|c|c|c|c|c|}
\hline & \multicolumn{9}{|c|}{ data transmission amount } & \multirow{2}{*}{$\begin{array}{c}\begin{array}{c}\text { data } \\
\text { reception } \\
\text { amount }\end{array} \\
\mathrm{M}\end{array}$} \\
\hline & (1) & (2) & (3) & (4) & (5) & (6) & (7) & (8) & (9) & \\
\hline T2002 & 4742.7 & 9798.8 & 13579.3 & 4317.6 & 13515.4 & 4742.9 & 4438.7 & 13263.4 & 19596.5 & 87995.3 \\
\hline T2003 & 4405.7 & 9232.3 & 13746.9 & 4224.5 & 12981.5 & 4767.4 & 4197.6 & 13234.6 & 19887.7 & 86678.2 \\
\hline T2004 & 4483.2 & 9639.9 & 13620.2 & 4638.2 & 13584.6 & 4460.5 & 4449.5 & 13909.7 & 19813.5 & 88599.3 \\
\hline T2006 & 4309.3 & 9185.1 & 13856.4 & 4301.4 & 13142.0 & 4874.5 & 4461.8 & 13581.1 & 19089.4 & 86801.0 \\
\hline T2007 & 4835.7 & 9317.9 & 13792.5 & 4167.3 & 13477.3 & 4471.4 & 4251.0 & 13855.9 & 20938.4 & 89107.4 \\
\hline T2008 & 4607.7 & 9513.9 & 13838.2 & 4184.9 & 13217.5 & 4359.7 & 4677.5 & 13601.2 & 19695.0 & 87695.7 \\
\hline T2009 & 4575.3 & 9282.7 & 13157.6 & 4009.0 & 13524.6 & 4796.6 & 4693.1 & 13124.4 & 20053.5 & 87217.0 \\
\hline T2010 & 4497.1 & 9651.8 & 13033.0 & 4592.2 & 13223.1 & 4666.2 & 4472.2 & 13077.9 & 19789.9 & 87003.4 \\
\hline
\end{tabular}

According to the results of codes and Table 6, it can be seen that the BP neural network algorithm can accurately predict the amount of the data transmission of the central node $\mathrm{M}$ within error permissibility.

\section{Conclusion and Future Work}

In this article, the CMST model is used to optimize the topology of the monitoring network of TengJiachong tailings reservoir. Then, the BP neural network algorithm is applied to predict the safety monitoring data to intelligently discover the faults of the tailings monitoring points. After the experiments and two-years stable operation of the system, it can be proved that the improved network topology has lower cost, more stable network transmission and less affect by the wild environment. In summary, the intelligent discovery algorithm proposed in this paper can predict the value of safety monitoring data more accurately and achieve better results.

However, the learning efficiency of BP neural network is fixed, the convergence speed of the network algorithm is slow. In the future, our work will focus on the optimization of BP neural network algorithm to improve the convergence rate and the efficiency of faults diagnosis.

\section{Acknowledgements}

The authors are grateful to the area editor and the anonymous reviewers for their valuable comments and suggestions on this paper. This work was a provincial and ministerial level vertical project under Grant No. 2012056068, and conducted by China University of Geosciences.

\section{References}

1. S. Y. Dong and Q. M. Li, "Discussion on Structure and Function Realization of Digital Tailings Ponds System," Journal of Safety Science and Technology, vol. 11, no. 5, pp. 78-83, May 2015

2. Y. Z. Gao, Y. Chu, and W. Liang, "Remote Sensing Monitoring and Analysis of Tailings Ponds in the Ore Concentration Area of Heilongjiang Province," Remote Sensing for Land and Resources, vol. 27, no. 1, pp. 160-163, January 2015 
3. D. C. Huang and S. C. Xie, "A Way to Predict the Settlement of Tailings Dam Based on BP Neural Network," Engineering of Surveying and Mapping, vol. 25, no. 8, pp. 53-56, August 2016

4. L. Huang, F. Miao, and M. X. Wang, "Designing and Setting up A Monitoring and Early Warning System for Tailings Ponds in A Region," China Safety Science Journal, vol. 23, no. 12, pp. 146-152, December 2013

5. Q. S. Huang, Q. Li, Y. J Wang, and J. Zhang, "Dry Beach of Tailings Dam Length Measurement Based on Waterline Recognition," Chongqing: IEEE Advanced Information Technology, Electronic and Automation Control Conference, pp. 503-507, December 2015

6. Y. Huang, Y. G. Li, and X. F. Chen, "Monitoring Information Processing for Saturation Line of Tailing Dam Based on DEKalman Filtering," Control Engineering of China, vol. 23, no. 9, pp. 1319-1324, September 2016

7. W. J. Liao, K. L. Huangfu, Y. He, and Z. Z. He, "Integrated Real-time Monitoring, Analyzing and Analyzing Pre-warning System for Tailings Reservoir," China Safety Science Journal, vol. 24, no. 8, pp. 158-163, August 2014

8. X. Li, J. Han, X. L. Lin, and X. D. Liu, "An Efficient Branch and Bound Algorithm for CMST Problem," Journal of Harbin Engineering University, vol. 28, no. 12, pp. 1371-1376, December 2007

9. M. Necsoiu and G. R. Walter, "Detection of Uranium Mill Tailings Settlement Using Satellite Based Radar Interferometry," Engineering Geology, vol. 197, no. 1, pp. 267-277, October 2015

10. S. G. Sun, P. Guo, Y. H. Zhang, and Z. Su, "Research on Safety of Fine Tailings Dam under the Influence of Saturation Line Distribution Characteristics," AER Advances in Engineering Research, vol. 44, no. 1, pp. 413-417, November 2016

11. J. Wang, Y. Q. Wen, Y. D. Gou, Z. Y. Ye, and H. Chen, "Fractional-order Gradient Descent Learning of BP Neural Networks with Caputo Derivative," Neural Networks, vol. 89, pp. 19-30, May 2017

12. L. G. Wang, F. W. Yang, and G. Z. Liu, "Application and Study on Vibrating Wire Sensor in Online Monitoring System of Tailings Reservoir," Nonferrous Metals(Mining Section), no. 3, pp. 83-86, June 2016

13. X. Y. Wan, L. H. Sun, H. M. Tian, Z. F. Huang, and H. Yang, "Research on 3S Integration Technology in the Tailings Pond Monitoring," International Conference on Energy, Environment and Sustainable Development. October 2011

14. T. L. Xu, X. Z. Lang, X. C. Pei, and L. X. Xue, "Research on Safety Monitoring and Early-warning System of Tailing Reservoir Based on Optical Fiber Transmission,” Gold, vol. 32, no. 7, pp. 43-47, July 2011

15. H. Zhang, Y. S. Zhao, and X. Li, "Research on Monitoring of Tailing Reservoirs Based on the Internet of Things-A Case Study of Huangmailing Phosphorus Chemical Tailing Reservoir," Safety and Environmental Engineering, vol. 22, no. 6, pp.143-150, December 2015

16. H. M. Zhou, Z. Q. Yuan, J. Su, and X. C. Yang, "The Present Situation and Prospects for Safety Online- Monitoring System of Tailings Pond," $3^{\text {rd }}$ International Conference on Mechatronics, Robotics and Automation, April 2015

17. L. F. Zhang, X. S. Liu, H. W. Wan, and X. Liu, "Luobei Graphite Mines Surrounding Ecological Environment Monitoring Based on High-resolution Satellite Data," Beijing: Conference on Multispectral, Hyper-spectral, and Ultra-spectral Remote Sensing Technology, Techniques and Applications V, November 2014

18. J. F. Zhang, Z. M. Qian, G. F. Ren, and S. S. Zhang, "Information Monitoring and Management System for Tailings Based on Lab VIEW," Wuhan: 3rd International Conference on Green Power, Materials and Manufacturing Technology and Applications, December 2013

19. W. Zhang, "Analysis on Public Safety Engineering Supervision Based on Internet of Things," Urban Construction Theory Research, vol. 316, no. 32, pp. 65-70, October 2013

Tianyong $\mathrm{Wu}$ is a $\mathrm{Ph}$. D. from the Faculty of Engineering, China University of Geosciences. His research interests include machine learning and neural network algorithm.

Chunyuan Zhang is a master student from the School of Computer Science, China University of Geosciences. Her research interests include neural network algorithm, remote sensing classification and recognition.

Yunsheng Zhao graduated from the Faculty of Engineering, China University of Geosciences, for the degree of Bachelor, Master and Ph. D. Now he is a professor of the China University of Geosciences, and the Director of the Department of Safety Engineering, China University of Geosciences, Wuhan, China. He is a senior member of Expert Group of National Safety Production Experts and the President of National Association of Safety Engineering Teaching Guidance Committee. His current research interests include system security theory and application, safety information engineering, and occupational safety and health. 